Estudios Geológicos, 66(2)

julio-diciembre 2010, 171-180

ISSN: $0367-0449$

doi:10.3989/egeol.40123.092

\title{
Interprétation des données magnétiques du chapeau de fer de Laachach (Jebilets centrales, Maroc): Implications minières
}

\author{
Interpretation of magnetic data of the Laachach gossan \\ (central Jebilets, Morocco): mining implications
}

\author{
M. Jaffal ${ }^{1}$, N. El Goumi ${ }^{1}$, M. Hibti ${ }^{1}$, A. Adama Dairou ${ }^{1}$, A. Kchikach ${ }^{1}$, A. Manar ${ }^{2}$
}

\begin{abstract}
RÉSUMÉ
Le massif hercynien des Jebilets centrales (Maroc) est caractérisé par l'affleurement de nombreux chapeaux de fer dont certains coiffent des amas sulfurés de grande importance économique (Kettara, Draa Sfar, etc.). Le présent travail porte sur l'interprétation de données magnétiques couvrant l'un de ces chapeaux de fer qui se situe près du village de Laachach à une trentaine de kilomètres au NordOuest de Marrakech. La carte magnétique du secteur étudié met en évidence plusieurs anomalies qui coïncident avec l'affleurement du chapeau de fer. Après réduction au pôle des données, l'analyse détaillée de ces anomalies permet de conclure que celles-ci seraient dues à des structures magnétiques allongées en direction subméridienne et décalées par une série de failles décrochantes dextres. Ces structures présentent généralement un pendage vers l'Ouest mais elles peuvent être localement subverticales. L'application de la déconvolution d'Euler aux données magnétiques de Laachach permet de calculer des solutions représentant ces structures dont la profondeur varie de 22 à $254 \mathrm{~m}$. L'interprétation quantitative des deux principales anomalies magnétiques mises en évidence au niveau de la zone d'étude a permis de caractériser la structure profonde des corps magnétiques de Laachach, qui pourraient correspondre à des amas sulfurés, compte tenu du contexte géologique et minier du secteur étudié. Ainsi, les deux corps modélisés constituent des cibles prioritaires de reconnaissance pour tout programme d'exploration minière susceptible d'être mené sur le site de Laachach.
\end{abstract}

Mots-clés: Laachach, Jebilets Centrales, Maroc, chapeau de fer, prospection magnétique.

\begin{abstract}
In the hercynian massif of Central Jebilets (Morocco), outcrop a large number of gossans which sometimes top economical orebodies (Kettara, Draa Sfar, etc.). The present study is devoted to the interpretation of magnetic data covering one of these iron hats, located near of the Laachach village, at about thirty kilometres north-westward of Marrakech. The magnetic map of Laachach highlights several anomalies which coincide with the outcrop of the gossan. Detailed analysis of reduced to the pole data allows us to conclude that these anomalies may be due to submeridian magnetic structures cut by a set of dextral transverse faults. These structures are generally dipping westward but they can be locally subvertical. The Euler deconvolution of the magnetic data gives moderately deeping solutions (22 to $254 \mathrm{~m}$ ). The quantitative interpretation of the two principal magnetic anomalies highlighted in the study area lead to better characterising of the deep structure of the Laachach magnetic bodies, that may correspond to massive sulphide occurrences, according to the geological and mining context of the study area. The two modelled bodies constitute priority recognition targets for any mining exploration program to be carried out on the Laachach site.
\end{abstract}

Keywords: Laachach, Central Jebilets, Morocco, gossans, magnetic prospecting.

\footnotetext{
1 Université Cadi Ayyad, Faculté des Sciences et Techniques, Département des Sciences de la Terre, Laboratoire de Géoressources. BP. 549, Marrakech, Maroc. Email: jaffal_m@yahoo.fr

2 Ministère de l'Energie et des Mines, Rabat, Maroc.
} 


\section{Introduction}

Le socle hercynien de la région de Marrakech est connu pour son potentiel minier, attesté par les nombreux gisements métallifères qu'il abrite (Draa Sfar, Kettara, Koudiat Aïcha, etc.) (fig. 1A). Ce socle qui affleure sous forme de massifs paléozoïques (Jebilets, Guemassa), a fait l'objet de nombreuses études géologiques académiques et de divers types de travaux d'exploration minière (Felenc et al., 1985; Mellal \& Maier, 1988; Hathouti, 1990; Hibti, 1993; Essaifi, 1995; Essaifi et al., 2003). Les chapeaux de fer, présents en abondance au niveau de ce socle, coiffent la plupart de ces gisements et constituent par conséquent d'excellents guides pour l'exploration minière. Dans le massif des Jebilets centrales, ces chapeaux de fer sont souvent organisés sous forme de linéaments orientés en direction sub-méridienne, parallèlement aux structures hercyniennes.

Des recherches minières sont actuellement menées dans ce massif en vue notamment de découvrir de nouveaux gisements et de mettre en évidence des extensions avales éventuelles de ceux déjà connus. Les cibles de l'exploration sont donc de plus en plus profondes et leur reconnaissance ne peut, a priori, se passer des méthodes géophysiques.

La présente étude concerne le chapeau de fer de Laachach qui se situe à une trentaine de kilomètres à vol d'oiseau au Nord-Ouest de Marrakech (fig. 1). Ce chapeau de fer constitue avec celui de Bouhane au Nord-Est et de Koudiat Aïcha au Sud, l'un des principaux linéaments d'amas sulfurés des Jebilets centrales.

Afin de préciser la structure profonde de ce chapeau de fer, on propose d'analyser les données magnétiques issues d'une couverture au sol de celui-ci. Le choix de cette méthode de prospection est justifié dans le caractère magnétique des amas sulfurés connus les gisements miniers du massif des Jebilets centrales (Draa Sfar, Kettara, Koudiat Aïcha). Le secteur concerné par cette étude est localisé sur la figure 1B en utilisant le système de coordonnées métriques de la projection Lambert du nord du Maroc (Zone 1).

\section{Contexte géologique et minier}

Le massif hercynien des Jebilets fait partie de la Meseta marocaine occidentale, il s'étend en direction E-W, au Nord de la ville de Marrakech, sur une longueur de $170 \mathrm{~km}$. Ce massif est constitué de trois grandes unités: occidentale, centrale et orientale, compte tenu de la nature des terrains et du style de la tectonique hercynienne qui l'affecte (Huvelin, 1977; El Hassani, 1980) (fig. 1A).

Le site de Laachach qui fait l'objet de la présente étude appartient à l'unité des Jebilets centrales, formée principalement, d'une série volcano-sédimentaire comportant des corps magmatiques intrusifs (gabbros, granites, etc.) dans des métapélites appelées «schistes de Sarhlef» dont l'âge est rapporté au Viséen supérieur-Namurien (Huvelin, 1977) (fig. 1A et B). L'indice de Laachach forme une série de chapeaux de fer de quelques kilomètres de long, parallèle à la direction générale des plans de schistosité (fig. 1B). Les chapeaux de fer sont constitués de bandes de schistes hématisés, dont la puissante varie de 10 à 20 mètres à l'affleurement, et comporte dans leur partie axiale une bande de limonite massive de 0,5 à 1,5 mètres de puissance (fig. 2 ).

D'un point de vue structural, les formations carbonifères qui constituent la série de Sarhlef des Jebilets centrales ont subies une déformation hercynienne intense. Elles ont d'abord été affectées par une phase majeure de plissement synschisteux de direction subméridienne (N0 à N30), accompagnée d'une schistosité $\mathrm{S} 1$ subparallèle à la stratification S0 et associée à un métamorphisme épizonal (Essaifi \& Hibti, 2008). Ensuite, durant une seconde phase, les Jebilets centrales ont été le siège de cisaillements sub-parallèles à la schistosité de flux $\mathrm{S} 1$, eux-mêmes repris par un plissement associé à une schistosité de crénulation S2. La troisième et dernière phase est caractérisée par des décrochements conjugués, d'ampleur variable, dextres N70 et senestres N135 (Bordonaro, 1983). Certains aspects de ces déformations sont visibles à l'échelle locale sur la carte géologique du secteur de Laacha$\mathrm{ch}$, en particulier la structuration subméridienne et les décrochements dextres N70 (figs. 1B et 2).

Sur le plan minier, le socle hercynien du massif des Jebilets centrales abrite de nombreux amas sulfurés dont certains constituent des gisements miniers de grande importance économique. Il s'agit majoritairement de corps minéralisés polymétalliques dominés par la pyrrhothine et intimement liées à un volcanisme rhyolitique à rhyo-dacitique (Hibti, 1993). Ces minéralisations sont concordantes avec la stratification des roches encaissantes et souvent associées à des zones de stockworks sous-jacentes. Ce sont des minéralisations de type volcano-sédimentaire qui présentent un caractère relativement distal par rapport aux manifestations 


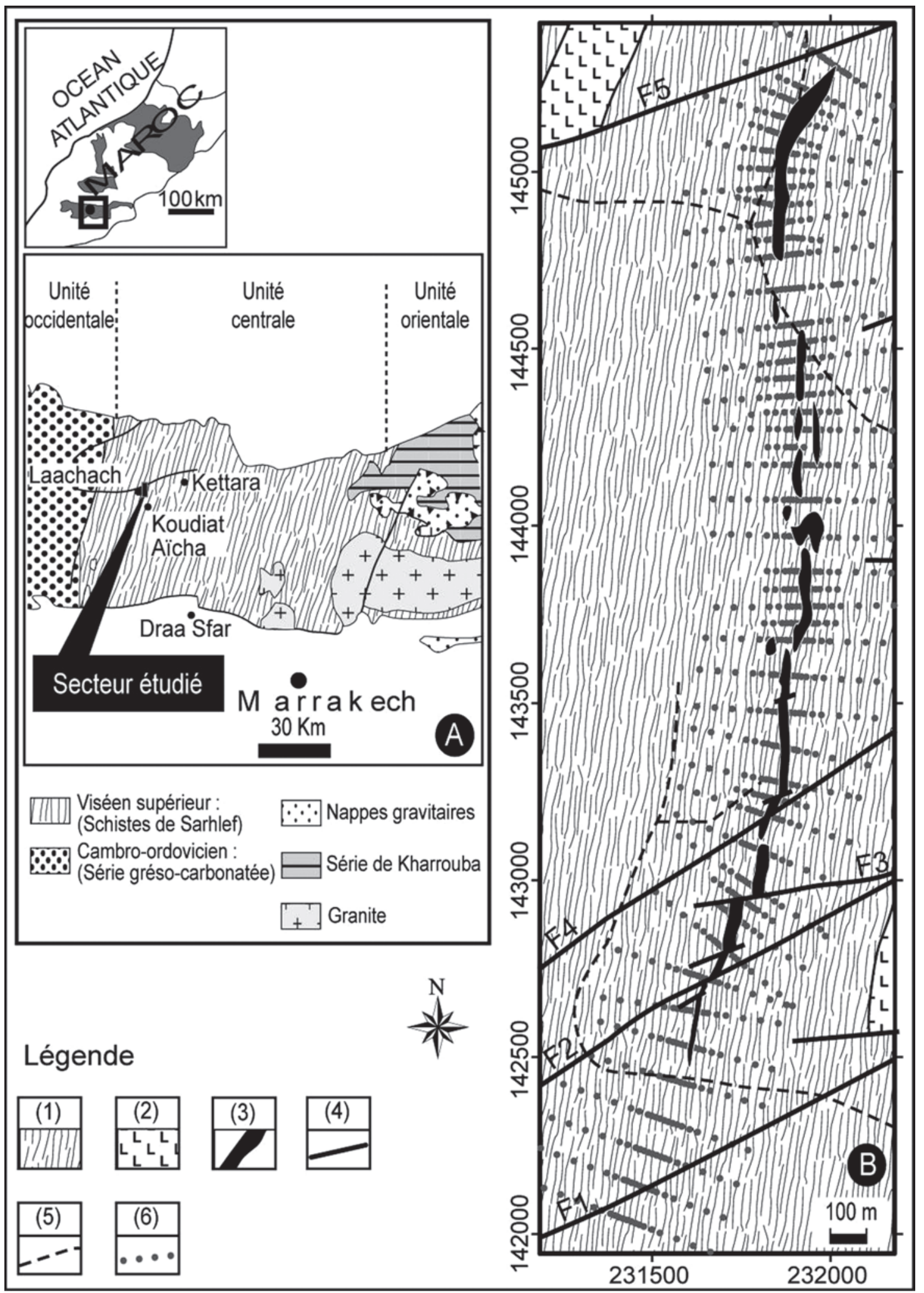

Fig. 1.-A) Situation générale du secteur de Laachach dans le massif des Jebilets centrales. B) Carte géologique schématique et profils du levé magnétique de la zone d'étude. 1. Schistes paléozoïques de Sarhlef; 2. Gabbro; 3. Chapeau de fer; 4. Faille; 5 . Talweg; 6. Stations des mesures magnétiques. 


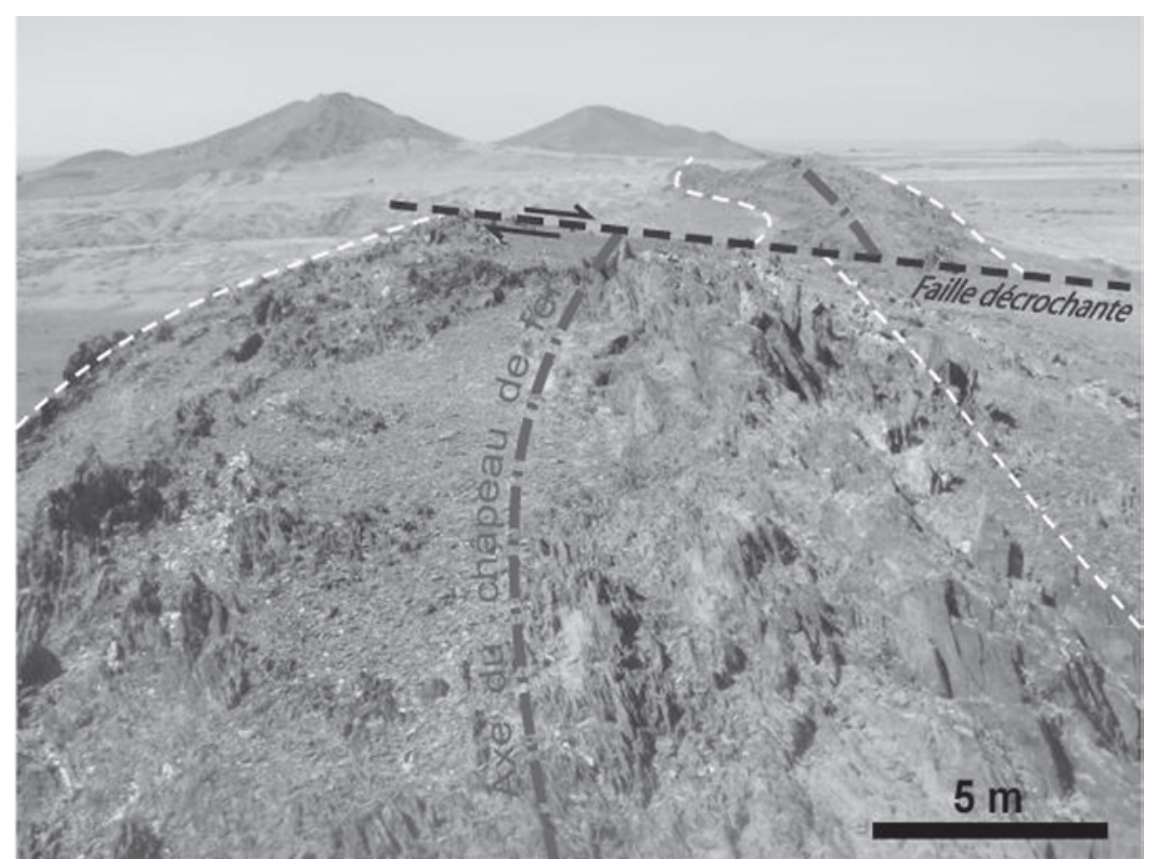

Fig. 2.-Vu d'ensemble du chapeau de fer de Laachach montrant le décalage de celui-ci par une faille décrochante dextre.

volcaniques contemporaines de leur mise en place (Bernard et al., 1988). Leurs caractéristiques minéralogiques et chimiques témoignent d'un environnement fortement réducteur entrainant la formation de paragenèse à pyrrhotine dominante. A l'affleurement, les minéralisations sulfurées sont fortement oxydées et altérées en produits limonitiques qui forment des chapeaux de fer. Ces derniers sont souvent organisés sous forme de linéaments de direction subméridienne (Bernard et al., 1988).

Felenc et al. (1986) proposent un modèle génétique dans lequel il envisage une mise en place de ces amas sulfurés lors d'une phase distensive au cours de laquelle se mettent en place des dépôts argilo-gréseux dans un bassin sédimentaire dont le développement est favorisé par le jeu de failles normales qui conduisent à une structuration en horsts et grabens. Cette distension est accompagnée par une importante activité magmatique caractérisée notamment par la mise en place d'un plutonisme bimodal (Essaifi et al., 2003). Ceci a conduit à de fortes perturbations thermiques ayant engendré le développement d'un hydrothermalisme qui se présenterait sous forme de cellules de convection affectant aussi bien les corps magmatiques que leur encaissant (Essaifi \& Hibti, 2008). Toutefois, la proximité des amas sulfurés (enrichis en métaux de base) et du plutonisme acide (appauvris en ces mêmes métaux) permet de considérer ce type de plutonisme comme principale zones source pour ce système hydrothermal (Essaifi \& Hibti, 2008). Les amas sulfurés des Jebilets centrales se seraient mis en place dans un environnement de rift épicontinenal de la zone externe de la chaîne hercynienne (Lescuyer et al., 1998)

\section{Données et méthode}

Les travaux d'exploitation dans les anciens gisements miniers du massif des Jebilets centrales (Kettara, Draa Sfar, etc.) ont permis d'effectuer des observations directes des minéralisations sulfurées et d'en étudier les caractéristiques minéralogiques et chimiques. Ainsi, ces minéralisations sont actuellement reconnues comme étant riches en pyrrhotine qui est un minéral magnétique. De ce fait, des anomalies magnétiques sont systématiquement associées à tous les gisements connus. D'où l'intérêt de la méthode de prospection magnétique dans la reconnaissance de ce type de gisements. Cette technique est d'ailleurs l'une des plus utilisées en exploration minière dans le massif des Jebilets centrales.

Les programmes de recherche minière dans ce massif ciblent prioritairement les chapeaux de fer dont celui de Laachach qui a fait l'objet d'une cou- 
verture magnétique par le service de la géophysique du ministère marocain de l'energie et des mines. Le levé magnétique a été effectué à l'aide d'un magnétomètre mesurant le champ total. Il a été mis en œuvre selon 48 profils de 500 mètres de long couvrant la totalité du chapeau de fer, avec une densité des stations plus serrée dans la zone médiane à l'approche de celui-ci (fig. 1B). Les données recueillies durant ce levé ont fait l'objet de traitements mathématiques (réduction au pôle et la déconvolution d'Euler) effectuées à l'aide du logiciel Geosoft. Ces traitements ont eu lieu à l'institut fédéral de géosciences et des ressources naturelles (BGR) de Hanovre (Allemagne) qui dispose d'une licence de ce programme.

En effet, afin de faciliter l'interprétation de ces données, celles-ci ont d'abord été réduites au pôle en tenant compte des paramètres locaux du champ magnétique terrestre à savoir, une intensité de 40.000 $\mathrm{nT}$, une inclinaison de $45^{\circ}$ et une déclinaison de $-5^{\circ}$, déterminés en utilisant le modèle géomagnétique IGRF 1995. Ce traitement a pour objectif d'éliminer les distorsions des anomalies engendrées par l'inclinaison du champ magnétique terrestre. Il permet d'obtenir des anomalies dont le maximum est centré sur les sources magnétiques. La réduction au pôle des données magnétiques a été effectuée en considérant une aimantation uniquement induite de direction parallèle au champ magnétique terrestre actuel. L'aimantation rémanente a été négligée car des mesures au laboratoire de celle-ci sur des échantillons prélevées au niveau la mine de Koudiat Aïcha située sur le même alignement de chapeaux de fer et donc fort probablement sur la même structure minéralisée, ont révélé de faibles valeurs (tableau 1). Ces mesures ont été effectuées à l'aide d'un cryomagnétomètre (Model 760 SRM-RF-SQUID, 2G Enterprises).

Dans un deuxième temps, la déconvolution d'Euler a été appliquée aux données magnétiques de Laachach dans le but de mieux caractériser les sources magnétiques. En effet, cette méthode permet d'estimer l'emplacement et la profondeur des sources anomaliques en utilisant le champ magnétique et ses dérivées horizontales (selon $\mathrm{X}$ et $\mathrm{Y}$ ) et verticale (selon Z) (Thompson, 1982). Les contraintes géologiques sont imposées par l'utilisation d'un paramètre caractéristique de chaque type de source: il s'agit de l'indice structural qui définit le taux d'atténuation de l'anomalie magnétique en fonction de la distance et qui dépend de la géométrie de la source. Le succès de l'application de cette méthode repose en grande partie sur une bonne estimation de ce paramètre (Reid, 1995).
Tableau 1.-Mesures de l'aimantation rémanente obtenues sur quelques échantillons prélevés au niveau de la mine de Koudiat Aïcha

\begin{tabular}{cclrrr}
\hline Echantillon & $\mathrm{NRM}(\mathrm{mA} / \mathrm{m})$ & $\mathrm{k}(\mathrm{SI})$ & $\mathrm{D}\left(^{\circ}\right)$ & $\mathrm{I}\left(^{\circ}\right)$ & $\mathrm{Q}$ \\
\hline K1 & 421,3 & 0,018324 & 22,1 & 33,5 & 0,72 \\
K2 & 538,4 & 0,036234 & 341,4 & 26,1 & 0,47 \\
K3 & 211,3 & 0,10225 & 203,7 & 69,3 & 0,06 \\
K4 & 252,5 & 0,078254 & 283,0 & 81,4 & 0,10 \\
K5 & 124,1 & 0,008923 & 112,6 & $-9,5$ & 0,44 \\
K6 & 142,6 & 0,0593 & 331,5 & 25,4 & 0,08 \\
K7 & 234,6 & 0,10459 & 87,3 & 5,3 & 0,07 \\
\hline
\end{tabular}

NRM: Aimantation rémanente naturelle. k: Susceptibilité magnétique. D: Angle de déclinaison de l'aimantation rémanente. I: Angle d'inclinaison de l'aimantation rémanente. Q: Rapport de Koenigsberger.

En outre, afin de mieux connaître la structure profonde des sources responsables des anomalies magnétiques de Laachach, une interprétation quantitative de ces dernières a été réalisée selon les deux profils A et B, localisés sur les figures 3, 4 et 5 . Ces deux profils ont été choisis de façon à recouper perpendiculairement les deux principales anomalies au niveau de leur maxima. L'interprétation quantitative a été effectuée à l'aide d'une technique inverse en deux dimensions et demi (2.5D) (Lai, 1984; Mickus, 2008).

\section{Discussion des résultats}

Les données magnétiques de Laachach sont présentées sous forme d'une carte de contours qui montre que ce secteur est caractérisé par un relief magnétique très agité, représenté par une série d'anomalies oblongues, orientées en direction subméridienne (fig. 3). Celles-ci sont principalement localisées au niveau de la zone médiane de la grille de levé qui coöncide avec l'affleurement du chapeau de fer. Leur amplitude est variable, elle peut atteindre 250 nT. Ces anomalies sont définies par plusieurs profils (au moins trois). Par conséquent, nous avons la certitude qu'elles ne correspondent pas à des artéfacts de mesures ou d'interpolation mais qu'elles sont bien liées à des structures magnétiques.

La réduction au pôle des données magnétiques a permis d'obtenir une carte qui reflète le degré de concentration des minéraux magnétiques à l'échelle de la zone couverte par le levé (fig. 4). Cette carte a été superposée à celle de la géologie du secteur de Laachach. Son examen permet de se rendre compte que les linéaments mis en évidence représentent des structures magnétiques sub-méridiennes, décalées 


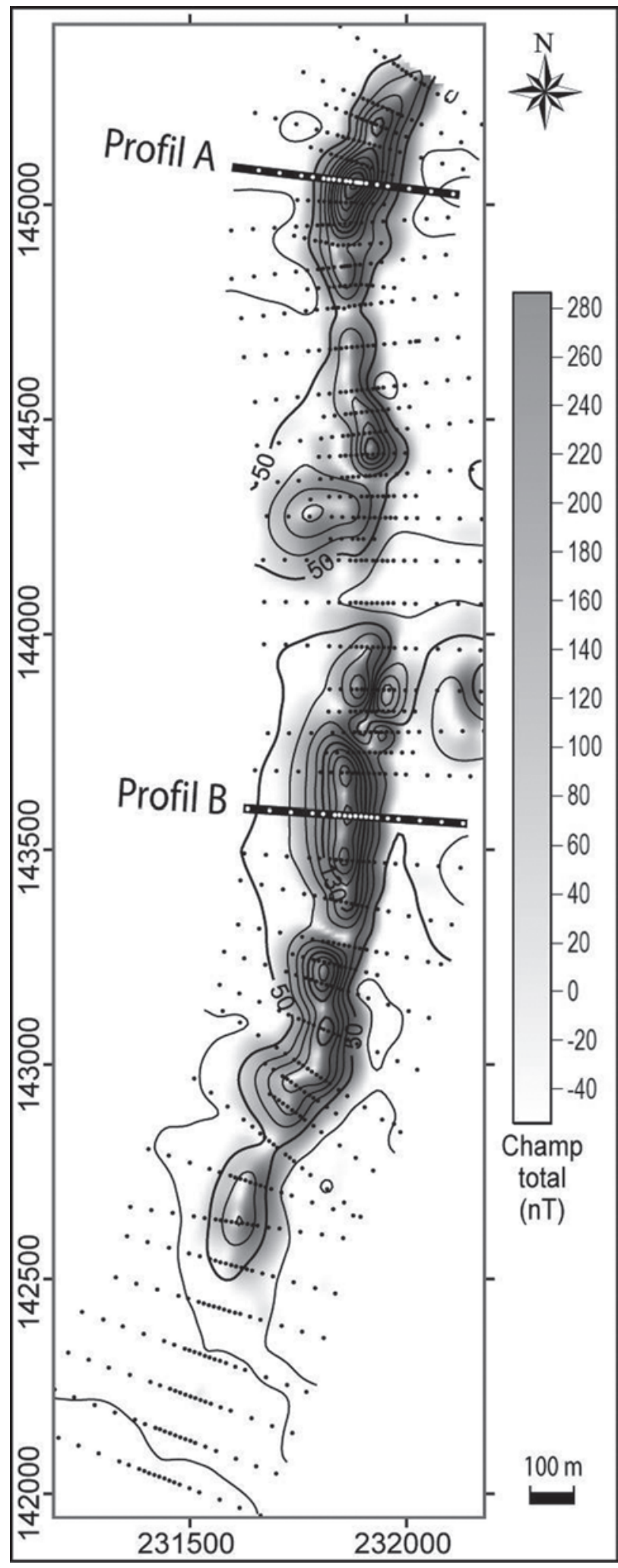

Fig. 3.-Contours des valeurs du champ magnétique total du secteur étudié. Un niveau de base de 40.400 nT a été soustrait aux données.

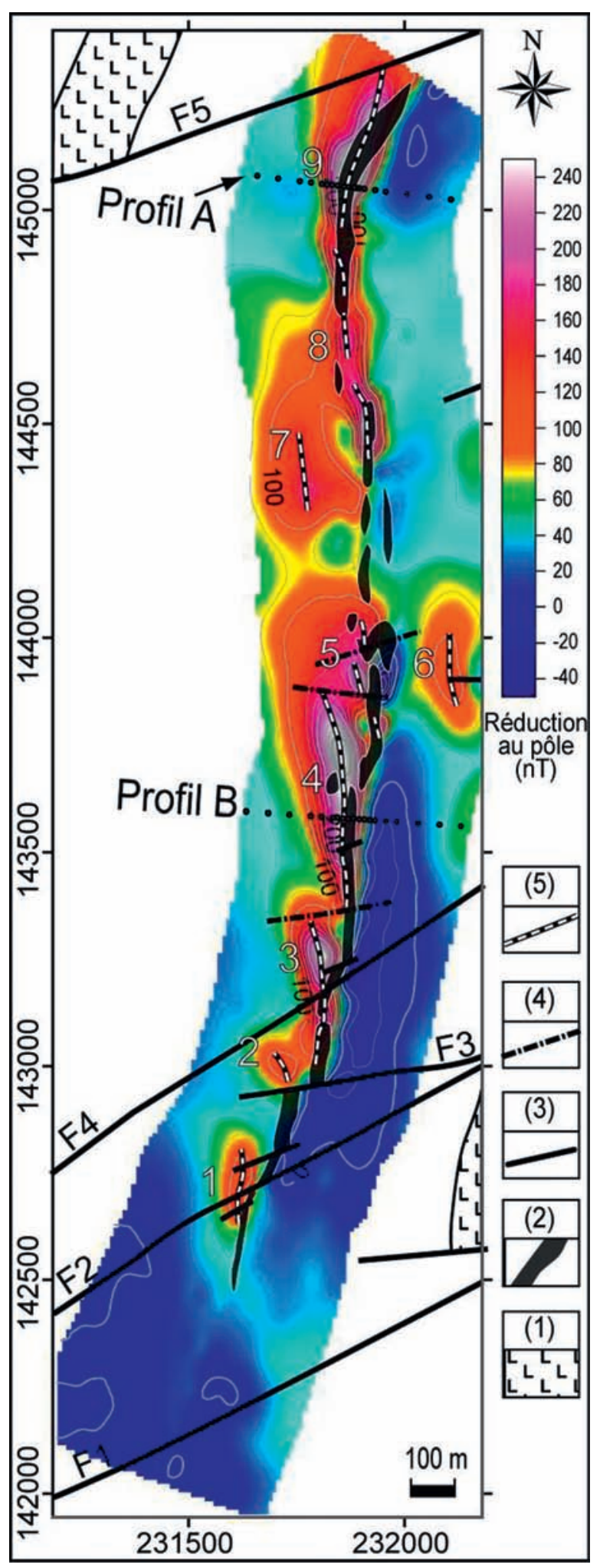

Fig. 4.-Contours des valeurs du champ magnétique total réduit au pôle, superposés au chapeau de fer de Laachach. 1. Gabbro; 2. Chapeau de fer; 3. Faille observée; 4. Faille interprétée; 5. Axe magnétique. 


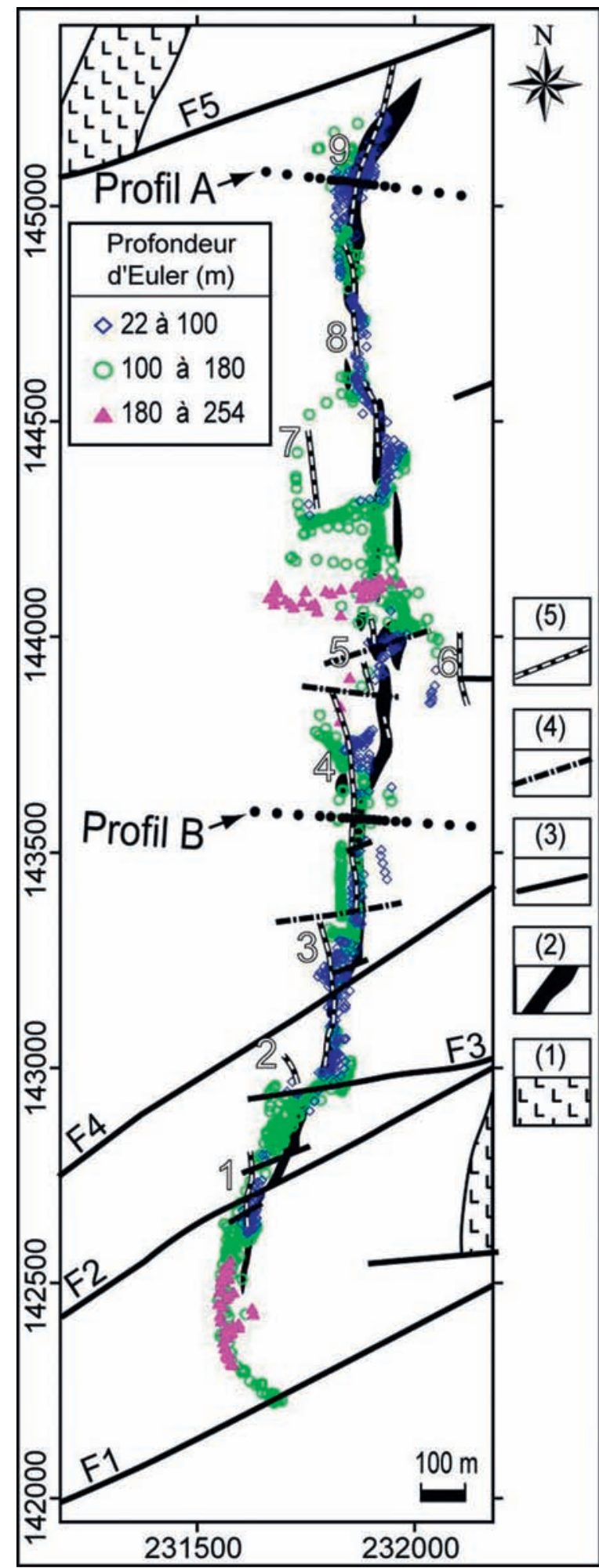

Fig. 5.- Solutions de la déconvolution d'Euler du secteur étudié. Les profondeurs d'Euler ont été calculées pour un indice structural IS=1, une taille de fenêtre de $10 \times 10$ et erreur relative maximale de $15 \%$. 1. Gabbro; 2. Chapeau de fer; 3. Faille observée; 4. Faille interprétée. par des failles transversales à jeux dextre. La forme très allongée de la plupart des anomalies représentent la signature magnétique de sources également allongées qui peuvent correspondre à des filons ou des lentilles comme c'est le cas de la plupart des gisements des Jebilets centrales (Kettara, Draa Sfar, Koudiat Aïcha). Par ailleurs, on constate que les anomalies magnétiques du champ total réduit au pôle du secteur de Laachach peuvent être subdivisées en deux types. Le premier regroupe des anomalies symétriques notées 6,7 et 8 , tandis que le second correspond à des anomalies asymétriques indiquées $1,2,3,4,5$ et 9 (fig. 4). Si on assimile les structures magnétiques responsables de ces anomalies à des corps de géométrie simple tels que des dykes ou des filons, ce qui est réaliste compte tenu des observations effectuées au niveau de la mine voisine de Koudiat Aïcha, on pourrait expliquer les différences de formes des anomalies par des variations de pendage de ces structures. En effet, compte tenu du fait que la géologie régionale du secteur d'étude est dominée par une structuration subverticale et que les travaux miniers dans le gisement de Koudiat Aïcha montrent que la minéralisation est en majorité à pendage ouest, on peut dire que les anomalies symétriques seraient dues à des corps subverticaux alors que celles asymétriques auraient pour origine des structures magnétiques inclinées vers l'Ouest.

La superposition des données géologiques et magnétiques permet également de se rendre compte que dans l'ensemble, le chapeau de fer apparait relativement bien jalonné par les anomalies (fig. 4). Cependant, dans le détail on constate que la convergence entre les deux types de données n'est pas parfaite pour plusieurs anomalies au niveau desquelles le chapeau de fer s'avère légèrement décalé vers l'Est. Ce décalage corrobore l'hypothèse de pendage vers l'Ouest qui impliquerait que le centre de masse de ces sources se situerait à l'Ouest des affleurements des chapeaux de fer et donc à l'aplomb des maxima des anomalies. Cependant, la coïncidence est meilleure entre le chapeau de fer et les signatures magnétiques dans les endroits où les sources magnétiques seraient plutôt subverticales. De même, on note que si la plupart des anomalies coïncident partiellement ou totalement avec le chapeau de fer de Laachach, il n'en est pas de même pour les anomalies 6 et 7 localisées en bordure la grille de levé, ce qui réduit leur chance d'être liées à des minéralisations sulfurées.

Compte tenu des informations géologiques disponibles sur les sources potentielles des anomalies magnétiques de Laachach, recueillies notamment au 

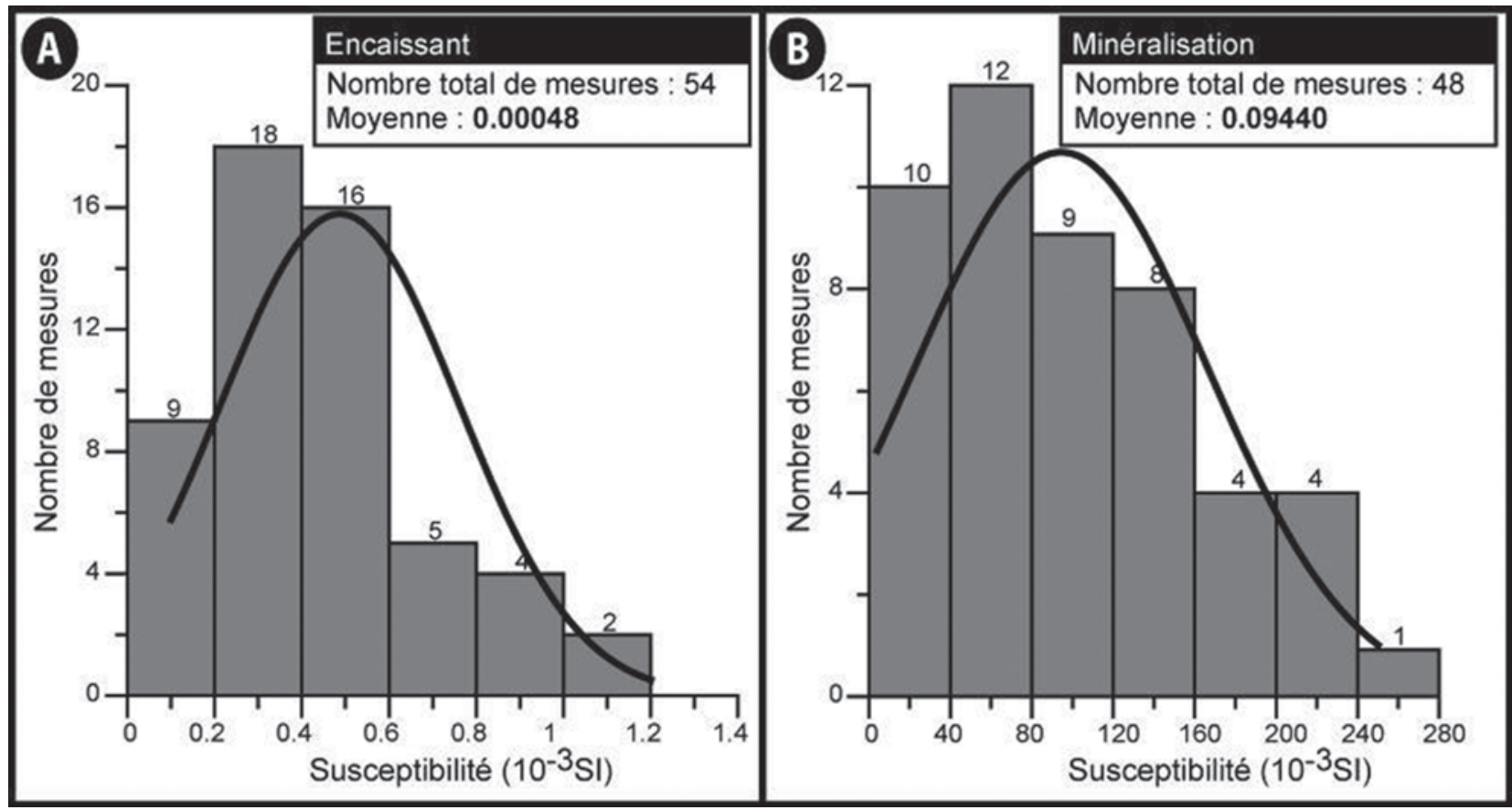

Fig. 6.-Susceptibilité magnétique relevées au Kappamètre.

niveau de la mine de Koudiat Aïcha, la technique de la déconvolution d'Euler a été appliquée aux données en considérant un modèle de dyke. Ce modèle s'avère le plus réaliste pour représenter la minéralisation sulfurée observée dans la mine précitée. En effet, cette minéralisation se présente sous forme d'amas ou de lentilles très étirés en direction N-S. Ainsi, les solutions d'Euler ont été calculées pour un indice structural (IS = 1) qui est la valeur attribuée au modèle de dyke dans le cas du magnétisme. Ce calcul a été effectué en utilisant une fenêtre de 10x10 mailles ou intervalles de la grille régulière calculée par interpolation des données magnétiques et une erreur relative maximale de $15 \%$.

Les profondeurs obtenues sont relativement faibles (22 à $254 \mathrm{~m}$ ) mais elles demeurent globalement comparables à celles connues dans la mine de Koudiat Aïcha (fig. 5). On constate par ailleurs, une bonne convergence des solutions calculées et leur coïncidence avec la majeure partie du chapeau de fer. Ceci confirme que la valeur de l'indice structural a été bien choisie, sauf au niveau de la zone située entre les anomalies 5,6 et 7 où le modèle de dyke ne serait pas convenable. Les plus grandes profondeurs $(>100 \mathrm{~m})$ sont obtenues au niveau de cette zone et dans l'extrémité sud du chapeau de fer. Cependant, partout ailleurs, des solutions faiblement à moyennement profondes ont été obtenues.
L'analyse détaillée de la position des sources calculées montre que celles-ci sont souvent situées à l'Ouest du chapeau de fer, ce qui confirme encore le pendage vers l'Ouest des sources magnétiques.

L'interprétation quantitative des deux principales anomalies magnétiques du secteur de Laachach a été effectuée le long de profils A et B (figs. 3, 4 et 5). Pour mener à bien cette modélisation, un intérêt particulier a été accordé à la susceptibilité magnétique, compte tenu de son importance dans le calcul des modèles. Ainsi, des mesures de ce paramètre ont été effectuées in situ sur le socle hercynien dont les affleurements sont dominés par les schistes de Sarhlef en utilisant un kappamètre KT9. En ce qui concerne la minéralisation, nous avons utilisées des valeurs de la susceptibilité magnétique issues de mesures effectuées avec le même type de susceptibilimètre sur des carottes de forages et sur des parois des galeries du fond de la mine de Koudiat Aïcha. La représentation de ces données sous forme d'histogrammes et leur fit par une loi normale (ou gaussienne) a permis de déterminer une valeur moyenne de la susceptibilité magnétique de la minéralisation (0.0944 SI) et de son encaissant (0.00048 SI). Cette valeur moyenne correspond au maximum de la courbe de fit en cloche (fig. 6).

Les résultats de l'interprétation quantitative sont présentés sur la figure 7 . Les modèles obtenus illus- 
trent la structure profonde des sources magnétiques. Celui du profil A correspond à un corps verticale d'une vingtaine de mètre de puissance dont le sommet se situe à une profondeur de $20 \mathrm{~m}$ et qui s'enracine jusqu'à $320 \mathrm{~m}$. Par contre, la seconde coupe modélisée au niveau du profil $\mathrm{B}$ montre une structure magnétique inclinée vers l'Ouest dont la profondeur varie de 20 à $290 \mathrm{~m}$. Les différences de profondeur par rapport à celles des solutions d'Euler peuvent s'expliquer par le fait que la méthode de modélisation utilisée ne permet pas une bonne appréciation de la base des corps magnétiques mais elle donne une bonne idée sur la profondeur du toit du corps et sur son pendage.

Les informations quantitatives disponibles désormais sur les deux sources magnétiques des deux principales anomalies observées, permettent de définir deux cibles potentielles pour l'exploration minière dans le secteur de Laachach. En effet, compte tenu du contexte géologique régional et local (présence de chapeaux de fer, proximité de la mine de Koudiat Aïcha, etc.), il y a de fortes chances que ces sources enfouies soient des amas sulfurés.

Comme nous l'avons signalé auparavant, les gisements miniers connus dans la région de Marrakech sont tous associés à des anomalies magnétiques provoquées par des minéraux tels que la pyrrhotine ou la pyrite. Malgré leur faible importance économique, ces minéraux magnétiques présentent l'avantage de permettre la détection indirecte des minéralisations sulfurée polymétallique plus intéressante à laquelle ils sont associés. Partant, de ces considérations, on peut dire que les principales anomalies mises en évidence dans le cadre de la présente étude dans le secteur de Laachach constituent des zones d'intérêts pour la recherche des amas sulfurées. Toutefois, l'expérience montre que la province des GuemassaJebilets est caractérisée par l'existence de signatures magnétiques liées principalement à la présence de gabbros. Par conséquent, l'application d'autres méthodes géophysiques (qui ne sont pas à notre portée) permettrait d'apporter plus de précisions sur la nature des sources magnétiques. Parmi les méthodes préconisées dans des contextes similaires à celui de Laachach, on peut citer la gravimétrie ou l'électromagnétisme du fait que les amas sulfurés constituent des corps très denses et d'excellents conducteurs.

\section{Conclusion}

Le présent travail montre l'intérêt de l'utilisation de la prospection magnétique pour la recherche des

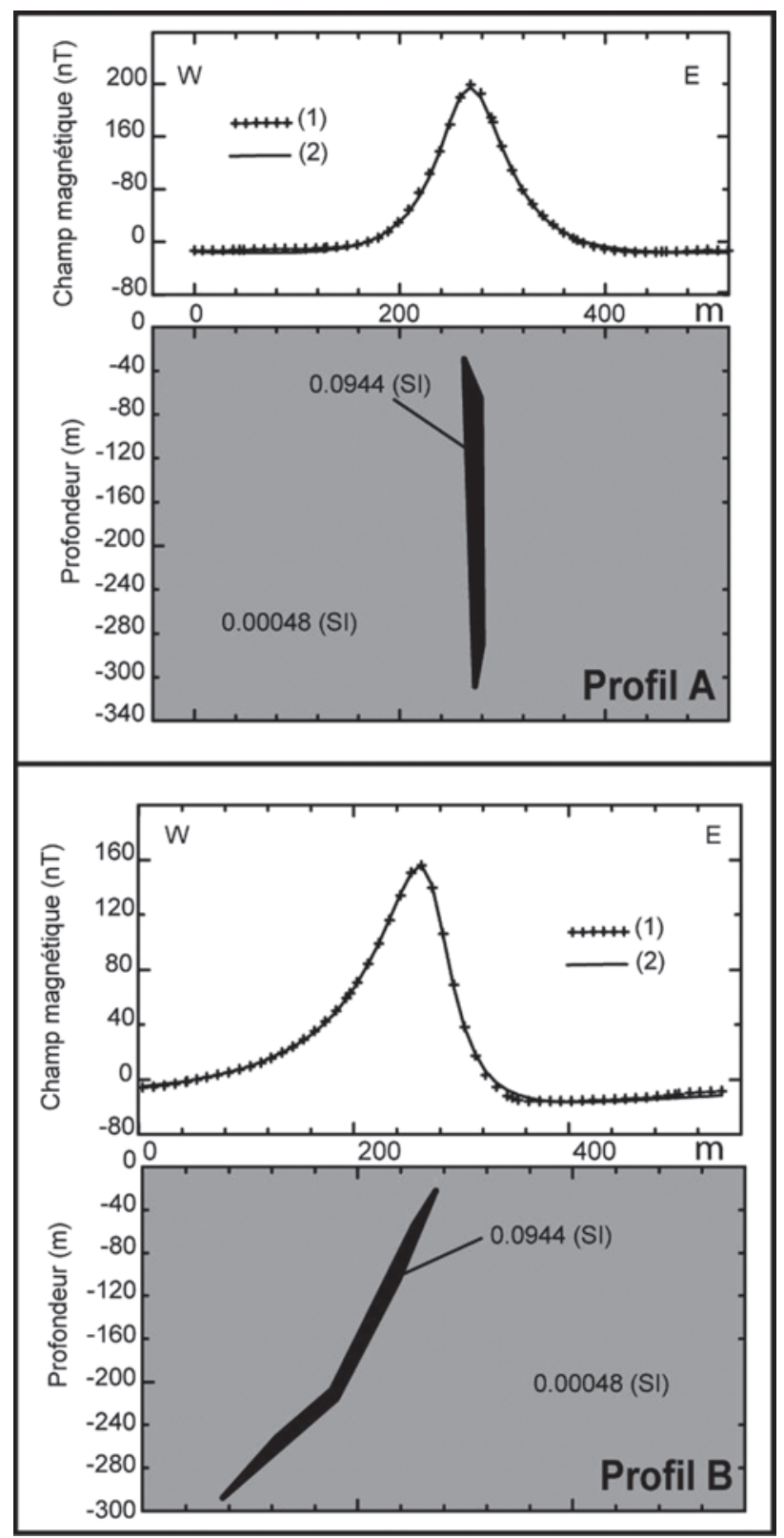

Fig. 7.-Modélisation 2.5D des anomalies magnétiques observées le long des profils $A$ et $B$ localisés sur les figures 3,4 et 5 . 1) Anomalie observée. 2) Anomalie calculée.

amas sulfurés dans du massif hercynien des Jebilets centrales. En effet, en se basant sur l'étude du secteur de Laachach. Les données magnétiques analysées révèlent l'existence d'anomalies associées aux chapeaux de fer qui affleurent dans ce secteur sur quelques kilomètres. L'interprétation qualitative et quantitative de ces anomalies permet d'apporter des précisions sur les caractéristiques des corps magnétiques qui en sont responsables. Il s'agit de struc- 
tures subméridiennes à pendage vers l'Ouest ou vertical qui correspondent très vraisemblablement à des amas de sulfures compte tenu du contexte géologique et minier de la zone d'étude.

\section{REMERCIEMENTS}

Cette étude a eu lieu dans le cadre de la préparation d'une thèse de doctorat financée par le Centre Nationale de la Recherche Scientifique et Technique (Bourse no: C03/008). Elle a été également réalisée dans le cadre du programme PROTARS II (Réf. P23/02) et de l'Action Intégrée Franco-Marocaine (Réf. MA/09/209).

Les auteurs tiennent à remercier le service de géophysique du ministère de l'Énergie et des Mines du Maroc (Rabat) pour les données magnétiques mises à leur disposition. Ils remercient aussi M. Cristoph Grissemann de l'institut fédéral de géosciences et des ressources naturelles (BGR) de Hanovre (Allemagne) pour sa collaboration.

Nos remerciements s'adressent également au Dr. M. Vicente Carlos Ruiz Martínez et à un lecteur anonyme pour leurs remarques et suggestions constructives.

\section{Références}

Bernard, A.J.; Maïer, O.W. \& Mellal, A. (1988). Aperçu sur les amas sulfurés massifs des Hercynides marocaines. Mineralium Deposita, 23: 104-144. doi:10.1007/BF00206659

Bordonaro, M. (1983). Tectonique et pétrographie du district à pyrrhotine de de Kettara. (Paléozö̈que des Jebilets, Maroc). Thèse de $3^{\text {ème }}$ cycle, Université Louis Pasteur, Strasbourg, France, 140 pp.

El Hassani, A. (1980). Etude lithostratigraphique, tectonique et pétrographique de la région de Sidi Bou-Othmane (Maroc). Contribution à la connaissance de l'évolution du segment hercynien des Jebilets centrales. Thèse $3^{\text {ème }}$ Cycle, Université Aix Marseille, Marseille, France, 114 pp.

Essaifi, A. (1995). Relations entre magmatisme-déformation et altération hydrothermale: l'exemple des Jebilets centrales (Hercynien, Maroc), Mémoires Géosciences Rennes, 66: 340 pp.

Essaifi, A.; Potrel, A.; Capdevilla, R. \& Lagarde, J.L. (2003). Datation U-Pb: âge de mise en place du magmatisme bimodal des Jebilets centrales (chaîne Varisque, Maroc). Implications géodynamiques. Comptes Rendus Geoscience, 335: 193-203. doi:10.1016/S1631-0713(03)00030-0

Essaifi, A. \& Hibti, M. (2008). The hydrothermal system of Central Jebilet (Variscan Belt, Morocco): A genetic association between bimodal plutonism and massive sulphide deposits ? Journal of African Earth Sciences, 50: 188-203. doi:10.1016/j.jafrearsci.2007.09.012

Felenc, J.; Alji, M.; Bellot, A.; Fournier, M.; Mheurras, M. \& Jidri, S. (1985). Découverte d'un amas sulfuré caché à pyrrhotite et métaux de base à Hajjar (Massif des Guemassa, Maroc). Chronique de Recherche Minière, 478: 61-66.

Felenc, J.; Fournier, M. \& Hmeuras, M. (1986). Contrôles géologiques des amas à pyrrhotine des Jebilets et Guemassa. Définition des guides de recherches. Rapport du Bureau de Recherches Géologiques et Minières, 86 MAR 131, 54 pp.

Hathouti, M. (1990). Etude gravimétrique et magnétique des amas sulfurés viséens de la région de Marrakech. $\mathrm{PhD}$ Thesis, Université des sciences et techniques du Languedoc, Centre géologique et géophysique, Montpellier, France, 206p.

Hibti, M. (1993). L'amas sulfuré de Hajjar: contexte géologique de mise en place et déformations superposées (Haouz de Marrakech, Meseta sud-occidentale, Maroc). Thèse de $3^{\text {ème }}$ cycle, Université de Marrakech, Morocco.

Huvelin, P. (1977). Etude géologique et gîtologique du massif hercynien des Jebilets (Maroc occidental). Note et Mémoires du Service Géologique du Maroc, 232 bis, 307 pp.

Lai, S.F. (1984). Generalized linear inversion of two and one-half dimensional gravity andmagnetic data. Ph.D. Thesis, University of Texas at Dallas, USA, $188 \mathrm{pp}$.

Lescuyer, J.L.; Leistel, J.M.; Marcoux, E.; Milési, J.P. \& Thiéblemont, D. (1998). Late Devonian-early Carboniferous peak sulphide mineralization in the Western Hercynides. Mineralium Deposita, 33: 208-220. doi:10.1007/s001260050141

Mellal, A. \& Maier, O.W. (1988). Recherches d'amas sulfurés dans la province des minéralisations syngénétiques volcano-sédimentaires hercyniennes: cas de Hajar dans le massif des Guemassa. Revue de l'Industrie Minérale, Mines et Carrières, Les Techniques, Mars-Avril, 121-131.

Mickus, K. (2008). Regional gravity analysis of Burkina Faso: Implications for the location of metallic ore deposits, Journal of African Earth Sciences, 50: 55-66. doi:10.1016/j.jafrearsci.2007.09.016

Reid, A.B. (1995). Euler deconvolution: Past, present and future - A review. 65th SEG meeting, Houston, USA, Expanded Abstracts, 272-273.

Thompson, D.T. (1982). EULDPTH - A technique for making computer-assisted depth estimates from magnetic data. Geophysics, 47: 31-37. doi:10.1190/1.1441278

Recibido el 6 de noviembre de 2009 Aceptado el 4 de junio de 2010 Publicado online el 9 de diciembre de 2010 